\title{
Planetary Exploration Using a Small Electromagnetic Sensor
}

\author{
Haoping Huang, Bill SanFilipo, and I. J. Won
}

\begin{abstract}
A prototype broadband electromagnetic (EM) sensor, GEM-5, has been built and tested as a possible new probe for the future Mars rover to seek an ice-bonded layer at a given depth below the Martian surface. The sensor, with a vertical coaxial coil configuration, will measure the terrain resistivity and susceptibility to determine lateral variations in resistivity and magnetic susceptibility. The lateral variations will indicate regions of resistivity/ susceptibility anomalies that may contain ice or water at depth. The forward solution for the sensor geometry over a layered formation and inverse algorithms to convert the EM data into the apparent susceptibility and resistivity are developed to investigate the ability of the sensor in detecting and resolving a buried (wet) ice layer in Mars-like geologic formations. Based on the simulated study, we find that the prototype sensor design should be able to resolve the lateral variations in resistivity/susceptibility under conditions of the Martian subsurface.
\end{abstract}

Index Terms-Vertical coaxial electromagnetic sensor, susceptibility and resistivity mapping.

\section{INTRODUCTION}

$\mathbf{S}$ HALLOW Martian geologic formations are permanently below the freezing point, and, therefore, any water bodies would be in permafrost similar to the polar region on the earth. The Martian atmosphere lacks water vapor in the midlatitudes, which leads us to believe that the ice has evaporated from shallow soils, and the permafrost condition may start deep in the equatorial region.

Past Mars missions have depended heavily on visual images. Sensors that have been tried (e.g., alpha-proton X-ray spectrometer and thermal emission spectrometer) do not see much deeper than a paper thickness below the surface. These sensors cannot sense through buried geologic formations that may contain water/ice lenses. In contrast, geophysical sensors can explore below the surface. On Earth, geophysical sensors are used for finding deep-buried oil and gas, mineral deposits, and groundwater formations, and they should work on Mars as well.

One of the difficulties in using current earth-bound geophysical sensors on Mars is the logistics, i.e., weight, size, and power consumption, as well as robustness and long-term stability. Additional factors may include maneuverability, autonomous operation, ground-contact requirements, and multiple sensor locations. This difficulty becomes obvious when faced with the physical sizes of Martian rovers.

Manuscript received August 18, 2004; revised February 22, 2005. This work was supported in part by the National Aeronautics and Space Administration, Jet Propulsion Laboratory, Pasadena, CA.

The authors are with Geophex Ltd., Raleigh, NC 27603 USA.

Digital Object Identifier 10.1109/TGRS.2005.846862
Geophysical exploration methods are broadly grouped into two categories, passive and active. A passive method senses the ambient field that is warped by hidden features; it includes gravity and magnetics. An active method uses a source that interrogates hidden features and measures their responses; it includes resistivity, electromagnetics (EM), seismics, and groundprobing radar (GPR).

Many factors influence the selection of geophysical methods. Generally, passive sensors are smaller, lighter, and simpler than active sensors. For active methods, the source and the receiver can be either colocated or separated. The former is compact and, thus, logistically suitable for rover-type applications, compared with the latter that require separated pieces of hardware. Another important factor is whether a sensor requires firm contact with the ground (e.g., resistivity, seismics) or not (e.g., EM, GPR); planting an array of geophones or electrodes into soil is not a trivial task for a small robotic rover.

The gravity method requires a mechanically and thermally stable platform, and its utility may be marginal for finding buried water/ice formations. Magnetics, convenient enough for the rover, can find ferrous minerals but may not do much good for exobiology or ice deposits. The resistivity method requires multiple electrodes to be planted into the ground and, thus, would be difficult for a single rover. Likewise, active seismic methods require an impact source and an array of geophones connected through multiple cables and, therefore, would not be possible for a single rover. Passive seismic devices (such as accelerometers) can detect Mars-quakes but are not useful for detecting water/ice lenses. GPR may be a viable sensor for very shallow exploration; however, it may entail excessive weight, high power consumption, and high data rate.

We believe an active EM sensor is most practical. It can be small and light enough for a rover and can measure lateral and vertical variations in resistivity and/or susceptibility, indicating regions of resistivity anomalies that may contain ice or water at depth. Such a method has been used for permafrost studies in cold regions [1]-[5].

In this paper, we propose a new EM sensor, GEM-5, that is specifically designed for a rover mission by keeping all above factors in mind, from the launch to deployment on Mars, autonomous operation, and extremely harsh environments. We also develop the analytical forward solution for the coil configuration over a layered formation, and the interpretation algorithms to invert the broadband data into apparent resistivity and susceptibility. Finally, we show two test examples on the earth. 


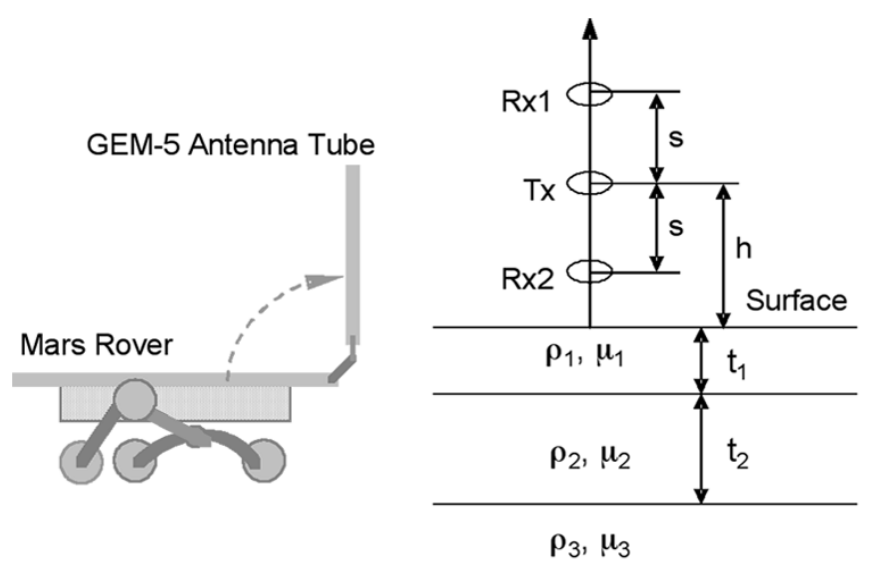

(a)

(b)

Fig. 1. (a) Scheme of the sensor installed on Mars rover and (b) over a layered half-space. The coil separation $s=35 \mathrm{~cm}$, and radius of the coils $a=3.35 \mathrm{~cm}$.

\section{PROTOTYPE SENSOR}

The sensor's operating principle is based on the GEM family of sensors [6], [7]. It will transmit a waveform containing multiple frequencies, and the receiver will measure the in-phase and quadrature components of the time-derivative of the secondary magnetic field. The sensing head, as shown in Fig. 1, consists of three coaxial coils mounted on a tubular structure that is envisioned to have a diameter of $7 \mathrm{~cm}$ and a length of $0.8 \mathrm{~m}$. The middle coil TX is the transmitter while the other two coils RX1 and RX2 are located at an equal distance from but on the opposite side of the TX. The upper coil RX1 is used as the system reference to monitor the primary field from the TX and RX2-RX1 as the signal channel. Since RX1 and RX2 are located at the same distance from the TX, the difference in output between RX1 and RX2 would be zero, or "balanced," in free space. When the sensor is brought to the ground, the balance breaks, producing a nonzero output that represents a contribution from the ground effect.

The digital, programmable sensor operates in the frequency domain at all user-selected frequencies continuously and simultaneously [6] and [7]. Each channel, denoted as RX1 reference and RX2-RX1 signal, receives, amplifies, and digitizes its output into a time-series lasting over a base period (1/30 s). The sensor has an analog-to-digital converter (ADC) rate of $192 \mathrm{kHz}$ and, therefore, each time-series has 6400 points per base period of $1 / 30$ th of a second (i.e., 192000/30). Both the signal and reference channels produce such a time-series at every base period. A digital signal processor (DSP) performs a sine and a cosine convolution at each frequency. The results from the signal channel are then normalized against those from the reference channel to produce the real or in-phase (I)-response and the imaginary or quadrature (Q)-response in a dimensionless unit of parts per million (ppm) as defined by

$$
\mathrm{ppm}=10^{6} \frac{\mathrm{RX} 2-\mathrm{RX} 1}{\mathrm{RX} 1} .
$$

The system stores a set of $I$ - and $Q$-data at all frequencies as the raw ppm data. Afterward, the two time-series are discarded. While the time-series processing goes on, the sensor now works
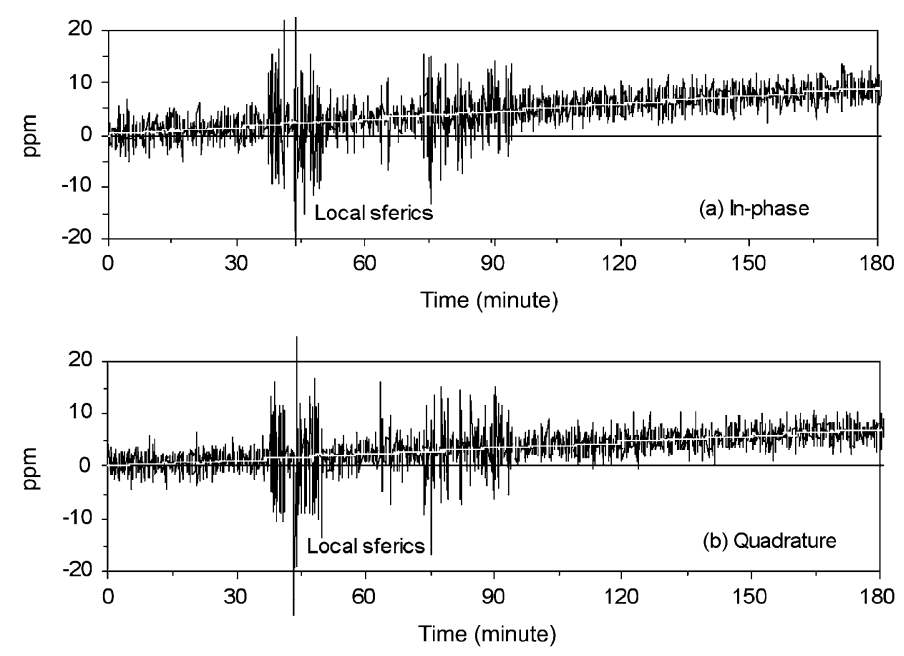

Fig. 2. Results from a static test. (a) $I$ - and (b) $Q$-component at $12 \mathrm{kHz}$ were recorded for $3 \mathrm{~h}$ after warming up.

for the next base period without interruption. The process repeats at every base period. Thus, the overall data rate is $30 \mathrm{~Hz}$ in this case, regardless of the number of frequencies used for the survey.

The measurement may be made as often as desired along the rover path. Such a gradiometer measurement had been tried by Barringer many years ago, but this work never appeared in the literature. Recently, more studies on this scheme have been performed [8]. The sensor, powered by a $12-\mathrm{V}$ battery, is featured by low power consumption $(<1 \mathrm{~W})$, lightweight $(<450 \mathrm{~g})$, and easy to deploy.

There are three main concerns in obtaining accurate datathe inherent noise level, the zero-level stability, and interference from the rover. In general, the gradiometer measurements are expected to have a low noise level due to the cancellation of the ambient noise recorded by the two receiver coils. However, the properties of the two receiver coils may not match perfectly, and their distances from the transmitter coil may not be exactly the same. The former causes residual noise in the data and the latter produces a constant zero-level shift. The EM noise is observed on the Earth largely due to the environmental EM noise including both natural and cultural EM noise [9]. Much of the natural EM field noise energy is from sferics caused by lightening discharges, and is not uniformly distributed throughout the sensor's bandwidth. They may be local $(<200 \mathrm{~km})$ or occur at great distances where propagation is efficient in the earthionosphere wave guide. The local discharges have a very sporadic and irregular nature, and the frequency of their occurrence depends strongly on local climatic and weather conditions. The distant discharges produce weaker noise, but they are more frequent and constitute a more or less steady noise background.

Fig. 2 shows the $I$ - and $Q$-components at $12 \mathrm{kHz}$ observed from a static test. The high amplitude noise is caused mainly by local sferics, while the noise envelope is produced by the distant sferics and the electronic noise. Most natural EM noise shows up as random noise, which may be removed by many techniques. For instance, stacking the data over $30 \mathrm{~s}$ at a sampling rate of $30 \mathrm{~Hz}$, the random noise should be decreased by a factor of $1 / \sqrt{ } 900$ or $0.033(30 \mathrm{db})$. The white curves in Fig. 2 represent 
the results of applying a median filter to the raw data. The noise levels (standard deviation) become $0.1 \mathrm{ppm}$ for $I$ and $0.08 \mathrm{ppm}$ for $Q$, reducing by a factor of $0.078(22 \mathrm{db})$ and $0.067(23 \mathrm{db})$, respectively.

The cultural EM noise can be both coherent and incoherent. There are a number of sources of cultural noise on the earth, e.g., power and phone lines, operating machinery, very low frequency transmissions, and so on. The cultural noise is time and location dependent, and occurs over the whole frequency band, but is strongest at power-line harmonics. The data in Fig. 2 contain some incoherent cultural noise, but the coherent noise is not obvious.

Fortunately, sferics do not exist on the Martian surface, and so the spikes and some background noise in Fig. 2 should not be observed. The cultural EM noise on Mars would be very low. The only source is the rover, which arises from two thingsmechanical motion between the sensor and metal components in the rover and mounting structure, and electromagnetic emissions from electronics in the rover (a combined effect of mechanical motion relative to dc current such as power in the rover is included). At this point, these are difficult to predict, and minimizing them is an engineering problem rather than a fundamental limitation.

The zero-level and its stability depend mainly upon the variations in temperature, equality of two receiver coils, and their distances from the transmitter coil. The constant residual zero-level shifts can be removed in the calibration procedures. Fig. 2 also shows drift behavior of the sensor for a 3 -h period after the sensor is warmed up.

The response to the rover is nominally part of the zero-level, but perturbations will result from small changes in the sensorrover geometry or movement of metallic parts on the rover. The coaxial coil configuration has a response null in the sensor equatorial plane, around which the rover could be located, and thus minimally coupled (note that the geometry portrayed in Fig. 1 is purely schematic and not meant to be the actual design). It would be difficult to avoid strong coupling to the rover with a conventional coplanar system. The multifrequency capability also provides a means of distinguishing between rover motion-induced noise and geologic responses of interest, since metal objects will respond well beyond the resistive limit and into the inductive regime in the frequency band we use.

\section{ElECtRomagnetic MODELS OF SHALlOW MARTIAN GEOLOGY}

Temperatures below the surface at the depths of interest are below the freezing point, so water will therefore be permafrost similar to the Earth's polar region. Because the Martian atmosphere lacks water vapor in midlatitudes, ice will have evaporated from shallow soils, and the permafrost condition may start deep in the equatorial region. Instruments on the Mariner 9, the Viking 1 and 2 missions, the Mars Pathfinder, the Global Surveyor, and, most recently, the twin rovers Spirit and Opportunity have provided quite a rich picture of both the Martian surface and atmosphere. The global images have shown a relatively dry surface with polar caps of water ice and dry ice, which vary with season.

There is ample evidence of water having flowed over the surface: it is criss-crossed with wadi-like riverbeds, bearing tes-
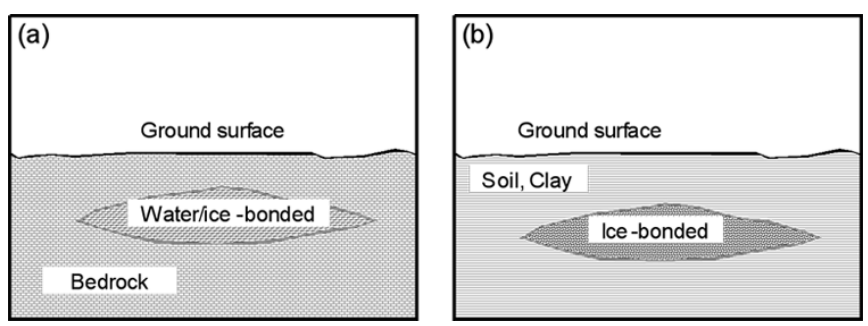

Fig. 3. Geologic models for water/ice-bonded formations on Mars.

timony to past violent water flows [10]-[12]. Estimates of the permafrost thickness range from $3-7 \mathrm{~km}$ near the poles to between 1-3 km near the equator [13]. Liquid water should exist under the permafrost, at least at midlatitudes. The depth to the top of the ice layer and the transition depth from permafrost to liquid water appear to have had significant effects on the morphology of surface features such as outflow channels, rampart craters, and terrain softening [11].

Our interest in this paper concentrates only on very near-surface EM properties of Martian geology, the first few meters from the surface. We are interested in mapping and sounding physical properties of soils and rocks, which may indicate the presence of shallow water/ice layers. If there are any water/icebearing lenses in sediments, and if the ice is partially melted due to dissolved minerals, then the lenses will have a much lower resistivity than that of the sediments [Fig. 3(a)]. Studies on permafrost terrain on the Earth indicate that the resistivity of frozen sediments, and/or ice-bonded sediments can vary from 20-1000 s of ohm-meter $(\Omega \cdot \mathrm{m})$, depending partially upon the volume percentage of ice [1]-[3]. The existence of ice may produce a resistivity contrast between the ice-bonded and dry sediments, which often produce measurable EM measurement anomalies, resulting in detection of an ice-bonded layer. Pure solid ice is an insulator; however, permafrost consists of small ice crystals in the pore spaces of the rock or soil matrix. These ice crystals usually have a thin liquid layer, and a thin liquid layer often surrounds the soil grains, particularly if some dissolved minerals are contained in the ice. Since the permafrost is not flushed with fresh rain or snow as on earth, the mineral content is expected to be relatively high. It is this small fraction of liquid that ultimately results in enhanced conductivity.

\section{RESPONSE OVER A HALF-SPACE}

Consider the sensor placed above a half-space as shown in Fig. 1. If a transmitter coil with a radius $a$ is placed at a height $h$ above the ground, the sensor's response can be derived based on (4.92) in [14], which is

$$
\begin{aligned}
\mathrm{RX} 2 & -\mathrm{RX} 1 \\
& =-I a \int_{0}^{\infty} R(\lambda) \lambda \exp (-2 \lambda h) \sinh (\lambda s) J_{1}(\lambda a) d \lambda
\end{aligned}
$$

where $I=\hat{I} e^{i \omega t}$ is the current in the transmitter coil in amperes, $s$ is the coil separation in meters, $\omega$ is the angular frequency, $J_{1}$ is the first-order Bessel function, and $R(\lambda)$ is

$$
R(\lambda)=\frac{Y_{1}-Y_{0}}{Y_{1}+Y_{0}}
$$



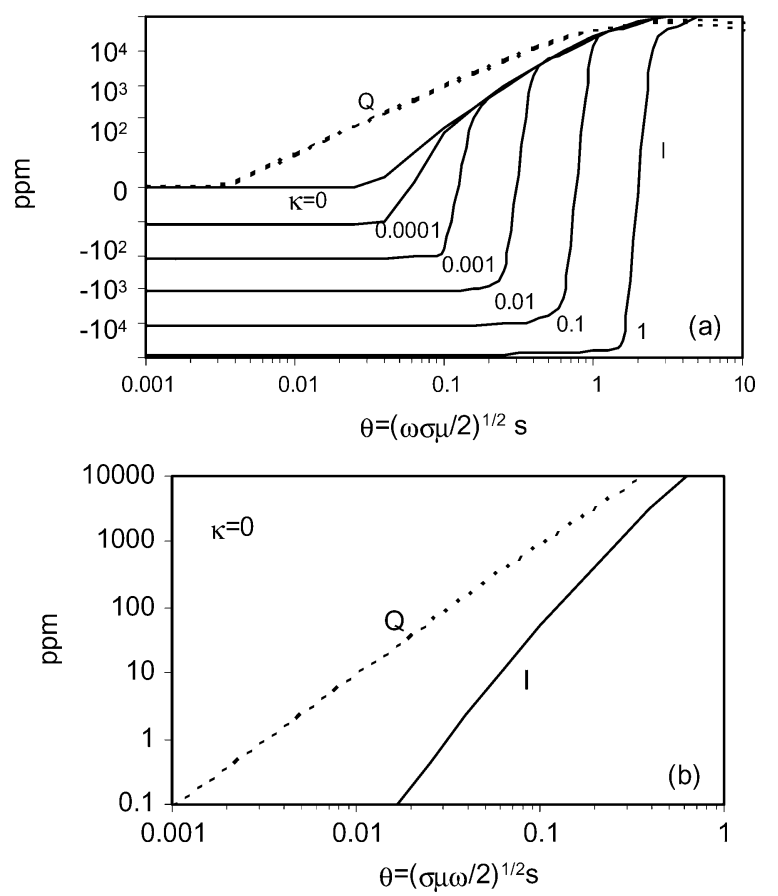

Fig. 4. (a) In-phase $(I)$ and quadrature $(Q)$ components of the secondary magnetic field of a homogeneous half-space as a function of induction number $\theta$ for various values of the susceptibility $\kappa$. (b) $I$ and $Q$-response at low induction numbers.

where $Y_{0}=u_{0} / i \omega \mu_{0}$ is the intrinsic admittance of free space, $Y_{1}$ is the surface admittance, $i$ is the imaginary number, $\mu_{0}$ is the magnetic permeability of free space, and $u_{0}$ is equal to $\left(\lambda^{2}+k_{0}^{2}\right)^{1 / 2}$ where $k_{0}$ is the free-space wavenumber. For an $L$-layer earth, $Y_{1}$ can be obtained by the following recurrence relation:

$$
Y_{l}=\hat{Y}_{l} \frac{Y_{l+1}+\hat{Y}_{l} \tanh \left(u_{l} t_{l}\right)}{\hat{Y}_{l}+Y_{l+1} \tanh \left(u_{l} t_{l}\right)}, \quad l=1,2, \ldots, L-1
$$

where $\hat{Y}_{l}=\left(u_{l}\right) /\left(i \omega \mu_{l}\right), u_{l}=\left(\lambda^{2}+k_{l}^{2}\right)^{1 / 2}$, and $k_{l}=\left(i \omega \sigma_{l} \mu_{l}-\omega^{2} \varepsilon_{0} \mu_{l}\right)^{1 / 2}$.

In the above equations, $t_{l}$ is the thickness, $\mu_{l}$ the magnetic permeability, and $\sigma_{l}$ the conductivity of $l$ th layer. $\varepsilon_{0}$ is the dielectric permittivity of free space. In the half-space at the bottom of the electrical section, $Y_{L}=\hat{Y}_{L}$.

The difference signal output is divided by RX1 as shown in (1), which is virtually the primary magnetic field

$$
\mathrm{RX} 1 \cong \frac{I a^{2}}{2\left(s^{2}+a^{2}\right)^{3 / 2}} .
$$

Substituting (2) and (5) into (1), we have

$$
\begin{aligned}
\frac{\mathrm{RX} 2-\mathrm{RX} 1}{\mathrm{RX} 1} & =-\frac{2\left(s^{2}+a^{2}\right)^{3 / 2}}{a} \\
\times & \int_{0}^{\infty} R(\lambda) \lambda \exp (-2 \lambda h) \sinh (\lambda s) J_{1}(\lambda a) d \lambda
\end{aligned}
$$

The in-phase $(I)$ - and quadrature $(Q)$-responses in ppm can be computed using (6). Fig. 4(a) shows the responses as functions of induction number $\theta=(\sigma \mu \omega / 2)^{1 / 2} s$ for various values of magnetic susceptibility $\kappa$ for the coil configuration with $a=$
$3.35 \mathrm{~cm}, s=35 \mathrm{~cm}$, and $h=0.45 \mathrm{~m}$ over a homogeneous half-space. At low induction numbers, the response is dominated by the magnetization effect, which is in-phase with, and in the same direction as, the primary field. This is the induced magnetization that occurs for an alternating magnetic field of a coil just as it does for the static magnetic field of the earth. At the other extreme, when $\theta$ is large, the induced conductive response overwhelms the magnetization effect. All curves in Fig. 4(a) tend to converge to that for $\kappa=0$ as $\theta \rightarrow \infty$. For midrange induction numbers, the magnetization effect and conductive effect are mixed. Martian soil or rock is expected to be resistive because it is frozen. Within the upper limit of the bandwidth of $96 \mathrm{kHz}$, the induction number range stays well below one $(\theta \ll 1)$. As shown in Fig. 4(b), both $I$ - and Q-responses in this range are small for nonmagnetic cases.

\section{APPARENT ReSistivity AND MAGNETIC SUSCEPTIBILITY MAPPING}

Resistivity/susceptibility mapping may outline lateral variations in electrical conductivity and magnetic susceptibility [15]. This will indicate regions of low or high resistivity that may contain water or ice below the surface. As shown in Fig. 4(a), the relative magnetic susceptibility $\kappa=\mu_{\mathrm{r}}-1$ can be determined from the in-phase response at low induction number and derived at the resistive limit $(\omega \sigma \rightarrow 0)$

$$
\kappa=\frac{-2 I}{I+10^{6} G} .
$$

$G$ is a geometry factor, and for this coil configuration, we have

$$
\begin{aligned}
G= & \left(a^{2}+s^{2}\right)^{3 / 2} \\
& \times\left\{\frac{1}{\left[(2 h-s)^{2}+a^{2}\right]^{3 / 2}}-\frac{1}{\left[(2 h+s)^{2}+a^{2}\right]^{3 / 2}}\right\} .
\end{aligned}
$$

If the subsurface were a truly homogeneous half-space, the magnetic susceptibility $\kappa$ obtained from (7) would be the true susceptibility; otherwise it would be the apparent susceptibility, often noted as $\kappa_{\mathrm{a}}$.

As shown in Fig. 4(b), the relationship between the induction number $\theta$ and quadrature response $Q$ can be approximately expressed using a simple empirical equation $\theta=10^{a+b Q}$ where $a=-2.48$ and $b=0.5$ for the sensor height of $0.45 \mathrm{~m}$. The resistivity $\rho$, the reciprocal of the conductivity $\sigma$, can be obtained for each individual frequency

$$
\rho=\frac{1}{\sigma}=\frac{\omega \mu_{0} h^{2}}{2 \theta^{2}} .
$$

If the subsurface is truly homogeneous, the resistivity obtained from (9) is the true resistivity. Otherwise, it would be the apparent resistivity $\rho_{a}$.

Then, we investigate theoretically the feasibility to detect shallowly buried ice/water-bearing lenses with the coil configuration in resistive environments. Two cases shown in Fig. 3 are considered. The first simulates an ice/water-bearing lens (200 $\Omega \cdot \mathrm{m}, \kappa=0$ ) sandwiched between more resistive rocks, e.g., alluvium and bedrock $(2000 \Omega \cdot \mathrm{m}, \kappa=0.0001)$. The second one simulates a resistive ice-bearing lens $(2000 \Omega \cdot \mathrm{m})$ in a less resistive clay layer $(200 \Omega \cdot \mathrm{m}, \kappa=0.00001)$. 

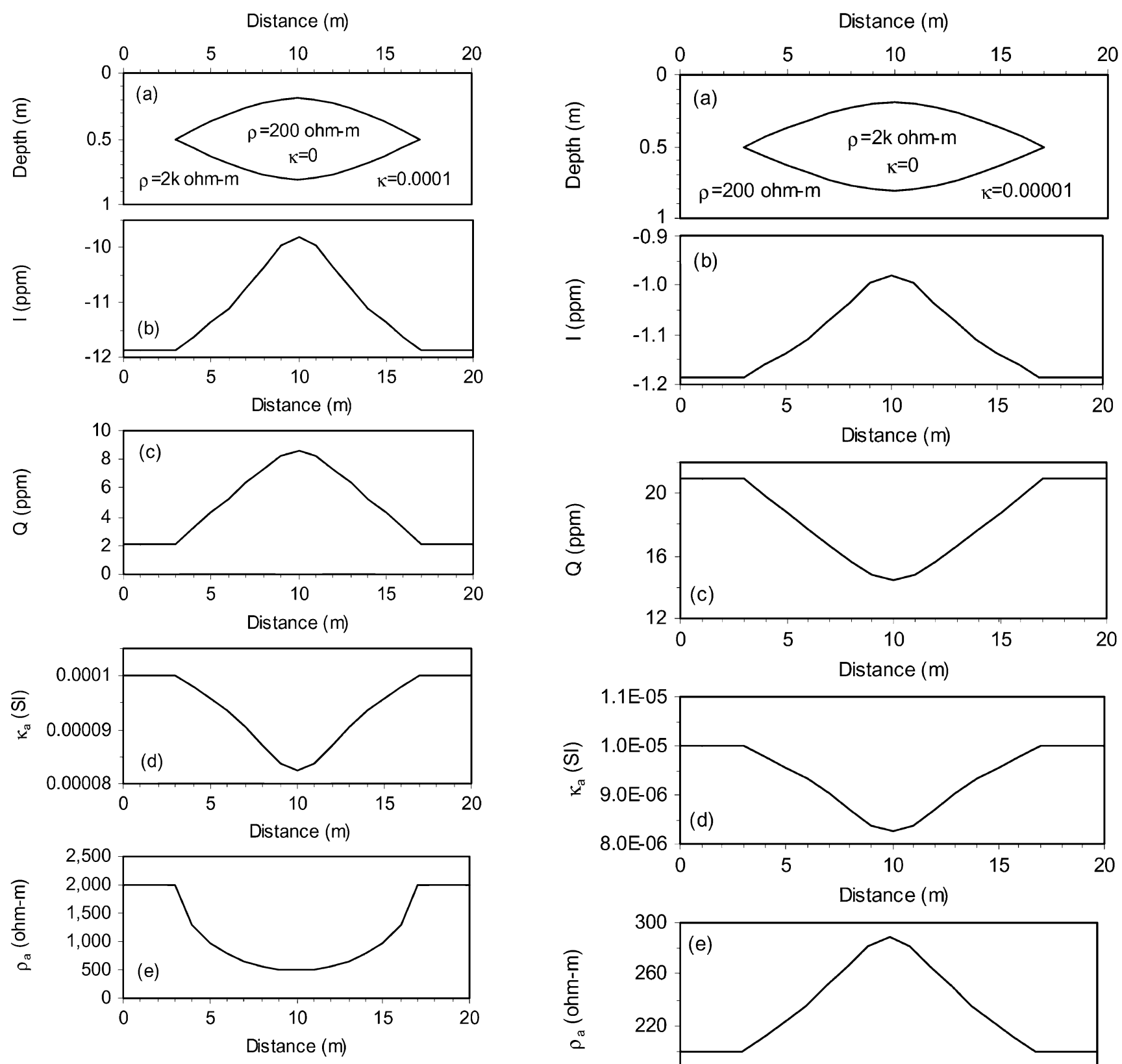

Fig. 5. (a) Model to simulate a case of less resistive lens sandwiched in more resistive host. (b) The $I$-response and (c) $Q$-response to the model and the apparent susceptibility (d) and resistivity (e).

The models, their EM responses, and the apparent susceptibility and resistivity are shown in Figs. 5 and 6. The EM data are computed from a series of one-dimensional models which are stitched together along a survey line. This approximation is justified because the small vertical coaxial configuration has very limited lateral coverage. Both targets produce anomalies in the EM data, apparent susceptibility, and resistivity. For example, the response amplitudes of background are about $-12 \mathrm{ppm}$ for $I$-component, and 2 ppm for $Q$-component as shown in Fig. 5(b) and (c), while the response amplitudes over the target become $-10 \mathrm{ppm}$ for $I$-component, and $8 \mathrm{ppm}$ for $Q$-component, resulting in an anomaly of $18 \%$ and $310 \%$, respectively. Also, the apparent susceptibility and resistivity show noticeable anomalies over the lens as illustrated in Fig. 5(d) and (e). The anom-

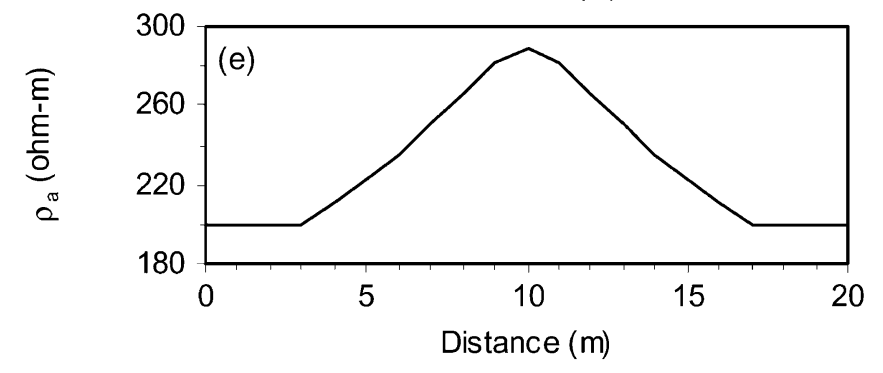

Fig. 6. (a) Model to simulate a case of more resistive lens sandwiched in less resistive host. (b) The $I$-response and (c) $Q$-response to the model and the apparent susceptibility (d) and resistivity (e).

alies in $Q$-component and resistivity become weaker when the target is more resistive than the host [Fig. 6(c) and (e)].

The depth of investigation (DI) in EM exploration is the most often stated issue and is of most interest, particularly for small EM sensors having negligible separation between the transmitter and receiver coils. The DI is related to many factors such as the sensor sensitivity, precision, operating frequencies, ambient noise level, target and host properties, and the techniques used in data processing and interpretation. Huang [16] developed a method to estimate the DI for a small EM sensor with horizontal coplanar coil-pairs. His results show that the DI 


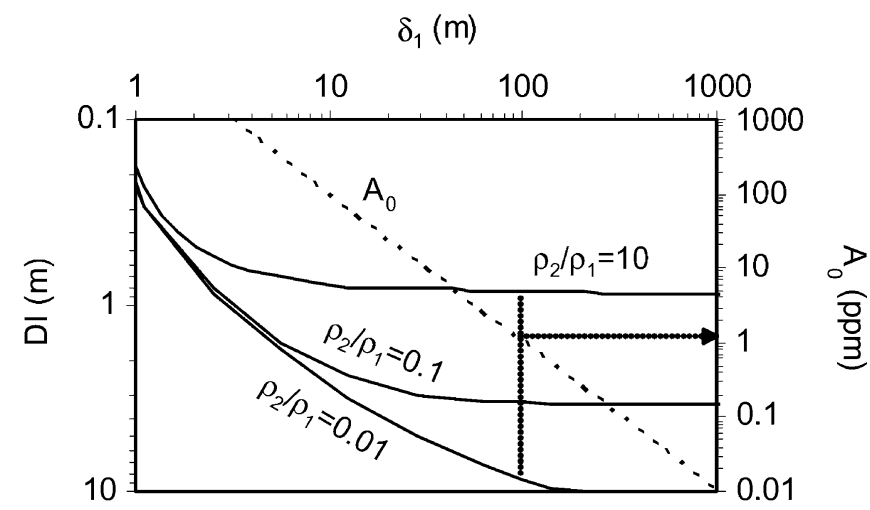

Fig. 7. Depth of investigation of the sensor as a function of the skin depth in the upper layer $\left(\delta_{1}\right)$ for resistivity contrasts $\left(\rho_{2} / \rho_{1}\right)$ of $0.01,0.1$, and 10 when the detection threshold is $10 \%$.

for a given detection threshold is approximately proportional to the square root of the skin depth in the host and conductivity contrast between the target and host. For a given skin depth, the DI increases with the target conductivity, conductivity contrast, and decreases with the detection threshold.

In this paper, we estimate the DI for the proposed sensor using the same method in [16]. Fig. 7 shows the DI as a function of the skin depth in the upper layer $\left(\delta_{1}\right)$ for resistivity contrasts $\left(\rho_{2} / \rho_{1}\right)$ of $0.01,0.1$, and 10 when the detection threshold is $10 \%$. Here, $\rho_{1}$ and $\rho_{2}$ are the resistivity of the upper layer and basement (target). The dashed line represents the background (upper layer) response amplitude $A_{0}$. This plot can be used as a graphic method to determine the DI for a given skin depth when the detection threshold is $10 \%$. As an example, consider the case when the skin depth in the overburden is $100 \mathrm{~m}$. This corresponds to DI $=3.3 \mathrm{~m}$ for a conductive target $\left(\rho_{2} / \rho_{1}=\right.$ $0.1)$, and DI $=0.83 \mathrm{~m}$ for a resistive target $\left(\rho_{2} / \rho_{1}=10\right)$. Since the background response $A_{0}=1.2 \mathrm{ppm}$ as shown by the dotted line in the figure, the estimate of DI is valid only when the total amplitude over the anomaly is greater than $1.32 \mathrm{ppm}$ or smaller than $1.08 \mathrm{ppm}(10 \%)$, and the $0.12 \mathrm{ppm}$ anomalous response must be reliably measured. If the resistivity contrast increases, the DI for conductive target will significantly increase with the skin depth in the upper layer. For example, the DI at the skin depth of $100 \mathrm{~m}$ will be up to $10 \mathrm{~m}$ for $\left(\rho_{2} / \rho_{1}=0.01\right)$.

It might be surprising to many readers that the DI of the small sensor for a detection threshold of $10 \%$ is up to $10 \mathrm{~m}$ for conductive targets. The key to reaching such a depth is that the sensor must have: 1) a low noise level; 2) a large dynamic range to accommodate near surface effects; and 3) a sufficient sensitivity to measure a small signal and to resolve the small changes in the signal. A sensor that can meet these requirements can be used for profiling, as well as depth sounding, in a variety of geological settings. The proposed sensor has been designed to satisfy these requirements. An argument on validity of a small sensor for depth sounding solely based on the induction number can be misleading. The frequency dependence does exist at the low induction numbers. The question is whether the sensor is able to resolve the small frequency dependency in the EM data.

Let us consider the situation shown in Figs. 3(a) or 5. We assume that the water/ice lens is thick enough to be treated as a two-layer model as shown in Fig. 8(a). The depth to the lower
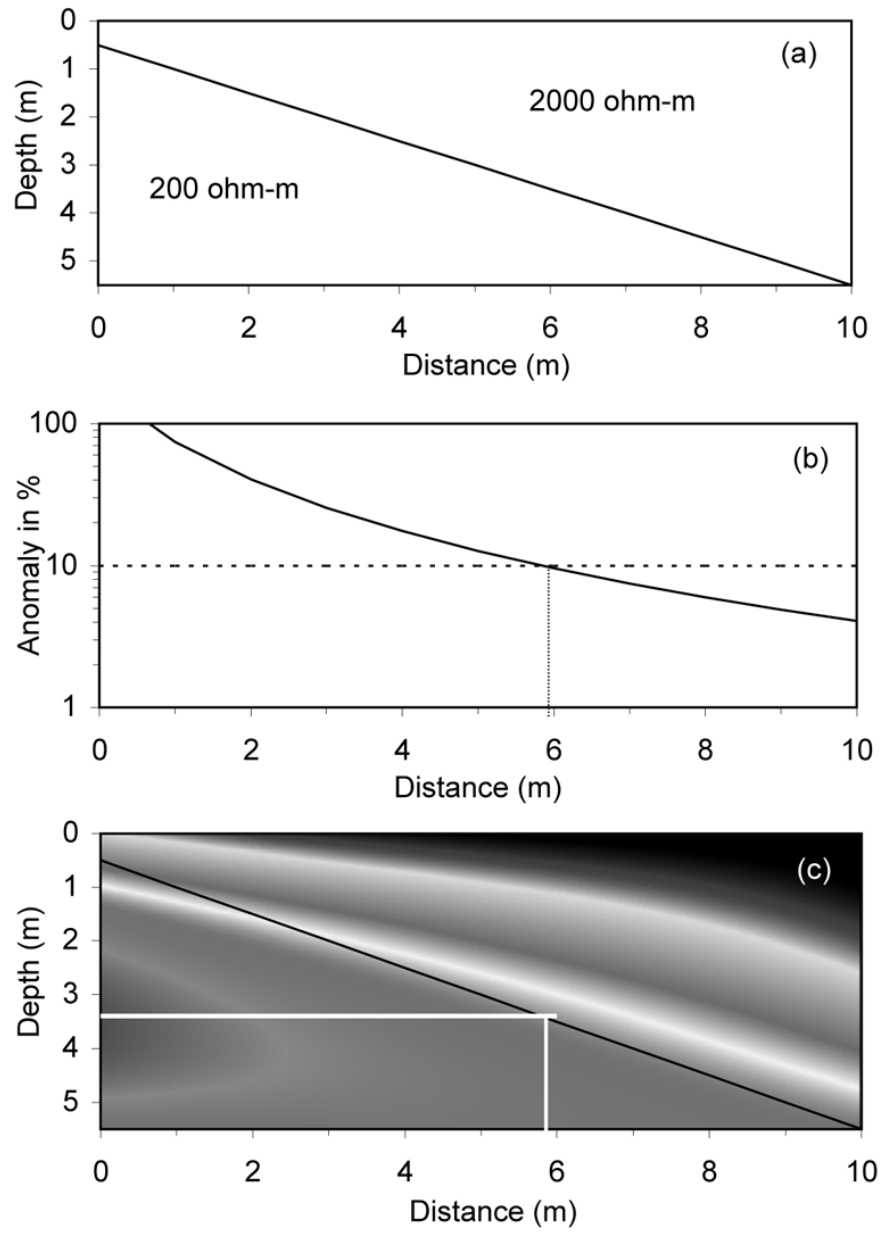

Fig. 8. (a) Two-layer section with the depth to the lower layer varying from $0.5 \mathrm{~m}$ on the left side to $5.5 \mathrm{~m}$ on the right side. (b) The anomaly at $10050 \mathrm{~Hz}$ for the two-layer model. (c) A two-dimensional section rendered by stitching a series of a one-dimensional model obtained from an inversion.

layer varies from $0.5 \mathrm{~m}$ on the left side to $5.5 \mathrm{~m}$ on the right side. The anomaly in percent at $10050 \mathrm{~Hz}$ from the model decreases with the depth to the lower layer. It is lower than $10 \%$ at about $6 \mathrm{~m}$ where the depth is about $3.4 \mathrm{~m}$. The skin depth in the upper layer is $225 \mathrm{~m}$ at $10050 \mathrm{~Hz}$, yielding a DI of $3.4 \mathrm{~m}$ (see the curve for $\rho_{2} / \rho_{1}=0.1$ in Fig. 7). If the sensor can measure an anomaly weaker than $10 \%$, the lower layer deeper than $3.4 \mathrm{~m}$ may be characterized. For example, Fig. 8(c) shows a two-dimensional resistivity section that is stitched together from a series of one-dimensional models obtained from an inversion [17] using noise-free data at four frequencies from $10-95 \mathrm{kHz}$. The model is mainly recovered in the interpreted resistivity-depth section, even at the rightmost side where the anomalies are from $4.3 \%$ at the lowest frequency to $3.8 \%$ at the highest frequency.

\section{FIELD TESTS}

The first field test was conducted in Geophex's backyard in Raleigh, NC. The soil is made of dense red clay with a susceptibility of 0.0005 (SI) and resistivity of about $90 \Omega \cdot \mathrm{m}$ [18]. The clay layer is thick enough to be treated as a homogeneous halfspace for this sensor. Over a spot where the ground is considered free of any buried clutter, we moved the mockup rover slowly over a distance of about $1 \mathrm{~m}$, which took about 2 min. Fig. 9(a) 

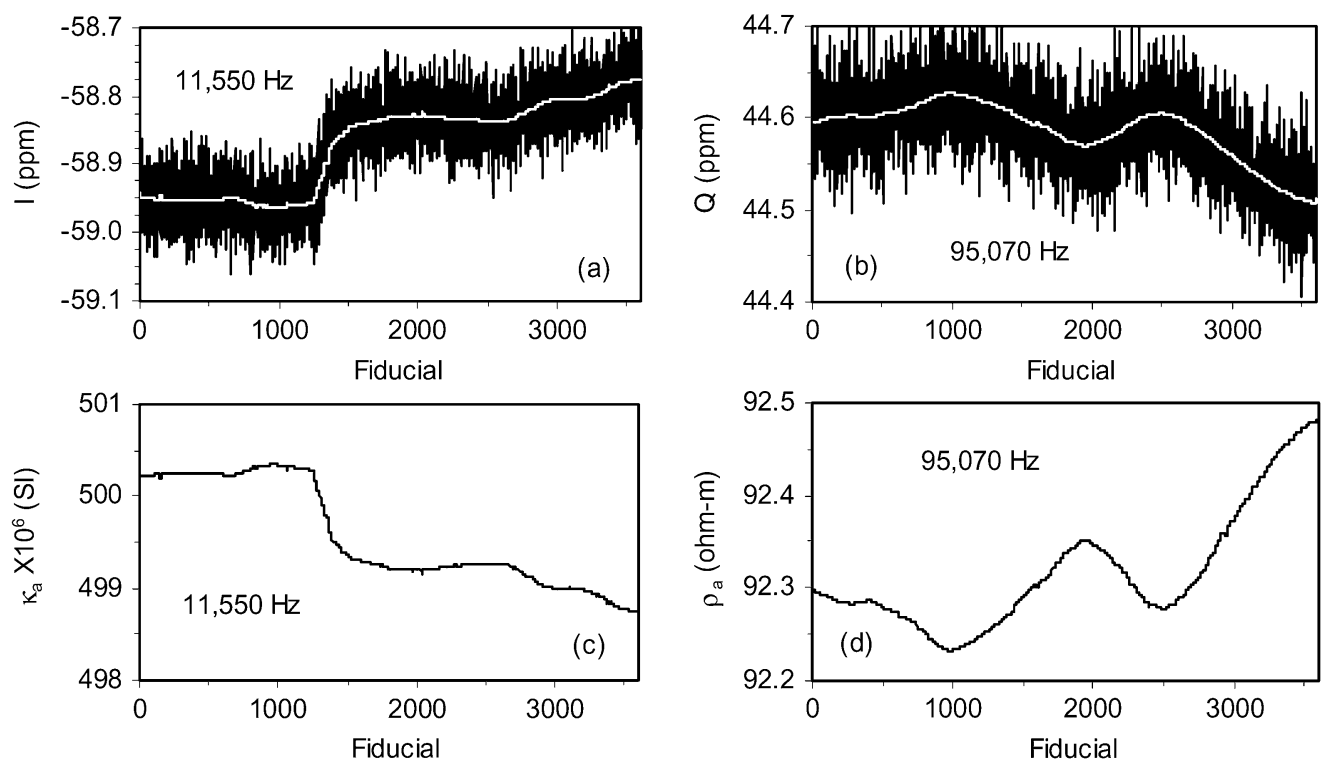

Fig. 9. (a) $I$-response at $11 \mathrm{kHz}$ and (b) $Q$-response at $95 \mathrm{kHz}$ obtained in Geophex's backyard. The raw data are shown in black, and the filtered data in white. (c) The interpreted magnetic susceptibility and (d) resistivity from the filtered data.
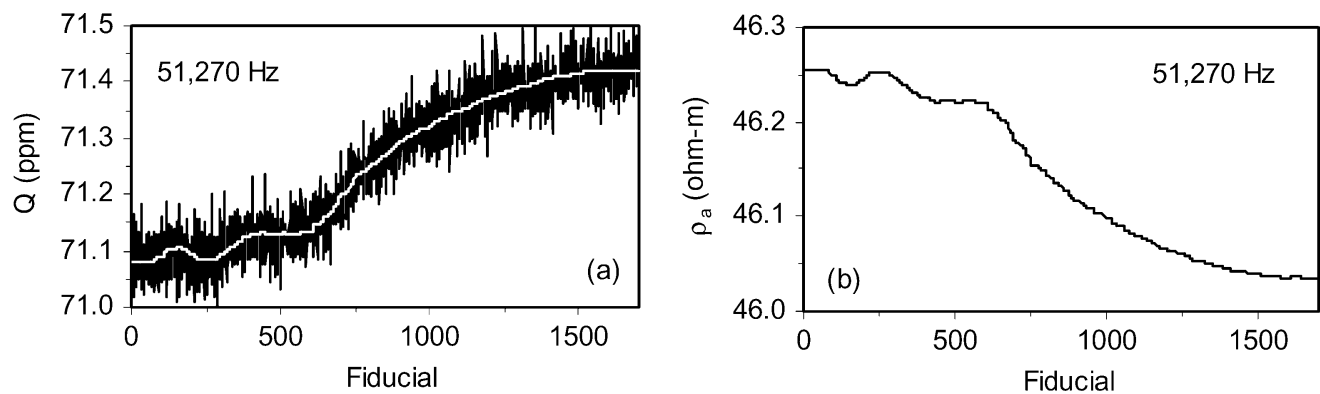

Fig. 10. (a) $Q$-response at $51270 \mathrm{~Hz}$ obtained at Lake Wheeler near Raleigh, NC. The raw data are shown in black, and the filtered data in white. (b) The interpreted apparent resistivity from the filtered data.

and (b) shows the measured $I$ - at $11550 \mathrm{~Hz}$ and $Q$-responses at $95070 \mathrm{~Hz}$ during this slow traverse. The measured $I$ - $Q$ data in parts per million are converted to produce the half-space susceptibility and resistivity profile shown in Fig. 9(c) and (d). The interpreted resistivity is centered on about $92.3 \Omega \cdot \mathrm{m}$, and magnetic susceptibility is about 0.0005 (SI). The test demonstrated that the sensor properly produced the expected range of the known ground susceptibility and resistivity.

The second test was at Lake Wheeler, a freshwater lake about ten miles south of downtown Raleigh, NC. The resistivity of freshwater varies from a few tens to hundreds of ohm-meter, depending upon its purity. We first placed the sensor in a floating plastic toolbox (the TX was $40 \mathrm{~cm}$ above the water level) and manually moved the box from an $80-\mathrm{cm}$ depth until the box went aground at the shoreline. Fig. 10(a) shows the $Q$-response at $51270 \mathrm{~Hz}$ over the lake, where the water is $0.8 \mathrm{~m}$ deep to the left and ends at the shore to the right. The responses increase very little from left to right, indicating the bottom resistivity is slightly lower than for the water. The apparent resistivity converted from the $Q$-response is about $46 \Omega \cdot \mathrm{m}$ as shown in Fig. 10(b).

It should be noted that the values of resistivity on the Martian surface are probably higher than those involved in the above examples. This would yield lower signal intensity. However, the EM noise level on the Martian surface is expected to be very low.

\section{CONCLUSION}

We investigated the ability of a vertical coaxial sensor in detecting buried water/ice-bonded layers in Mars-like geologic formations, formulated the forward solution for the sensor geometry over a layered formation and developed techniques to convert the EM data into the apparent susceptibility/ resistivity. The prototype sensor is specifically designed for a rover mission, from the launch to deployment on Mars, with autonomous operation and in extremely harsh environments. The gradiometer measurement provides low noise and a stable zero-level. Our modeling results show that the prototype sensor should be able to measure the resistivity and susceptibility of the Martian subsurface. Variations in resistivity/susceptibility may provide information on possible water/ice-bonded formations. Our testing results on the earth for the sensor's stability and accuracy, as well as geologic resolutions show that it can provide for possible planetary exploration. 


\section{REFERENCES}

[1] P. Hoekstra, P. V. Sellman, and A. Delaney, "Ground and airborne resistivity surveys of permafrost near Fairbanks Alaska," Geophysics, vol. 40, pp. 641-656, 1975.

[2] P. Hoekstra, "Electromagnetic methods for mapping shallow permafrost," Geophysics, vol. 43, pp. 782-787, 1978.

[3] B. J. Todd and S. R. Dallimore, "Electromagnetic and geological transect across permafrost terrain, Mackenzie River delta, Canada," Geophysics, vol. 63, pp. 1914-1924, 1998.

[4] W. J. Scott, P. V. Sellmann, and J. A. Hunter, "Geophysics in the study of permafrost," in Geotechnical and Environmental Geophysics, S. H. Ward, Ed. Tulsa, OK: Soc. Exploration Geophys., 1990, vol. 1, pp. 355-384.

[5] J. J. Daniels, G. V. Keller, and J. J. Jacobson, "Computer-assisted interpretation of electromagnetic soundings over a permafrost section," Geophysics, vol. 41, pp. 752-765, 1976.

[6] I. J. Won, D. Keiswetter, G. Fields, and L. Sutton, "GEM-2: A new multifrequency electromagnetic sensor," J. Environ. Eng. Geophys., vol. 1, no. 2, pp. 129-137, 1996.

[7] I. J. Won, "Small frequency-domain electromagnetic induction sensors," Leading Edge, vol. 22, pp. 320-322, 2003.

[8] D. Sattel and J. Macnae, "The feasibility of electromagnetic gradiometer measurements," Geophys. Prospecting, vol. 49, pp. 309-320, 2001.

[9] K. G. McCracken, J. P. Pik, and R. W. Harris, "Noise in EM exploration systems," Explor. Geophys., vol. 15, pp. 169-174, 1984.

[10] M. H. Carr, "Mars: A water-rich planet?," Icarus, vol. 56, pp. 187-216, 1986.

[11] V. R. Baker, The Channels of Mars. Austin: Univ. Texas Press, 1982.

[12] S. W. Squyres et al., "Ice in the Martian regolith," in Mars, H. H. Kieffer et al., Eds. Tucson: Univ. Arizona Press, 1992, pp. 523-554.

[13] F. P. Fanale, "Global distribution and migration of subsurface ice on Mars," Icarus, vol. 67, pp. 1-18, 1986.

[14] S. H. Ward and G. W. Hohmann, "Electromagnetic theory for geophysical applications," in Electromagnetic Methods in Applied Geophysics, M. N. Nabighian, Ed. Tulsa, OK: Soc. Exploration Geophys., 1988, vol. 1, Theory, pp. 130-311.

[15] H. Huang and I. J. Won, "Conductivity and susceptibility mapping using broadband electromagnetic sensors," J. Environ. Eng. Geophys., vol. 5, no. 4, pp. 31-41, 2000.

[16] H. Huang, "Depth of investigation for small broadband electromagnetic sensors," Geophysics, to be published.

[17] S. C. Constable, R. L. Parker, and C. G. Constable, "Occam's inversion-A practical algorithm for generating smooth models from electromagnetic sounding data," Geophysics, vol. 52, pp. 289-300, 1987.

[18] H. Huang and I. J. Won, "Automated anomaly picking from broadband electromagnetic data in UXO survey," Geophysics, vol. 68, pp. 1870-1876, 2003.

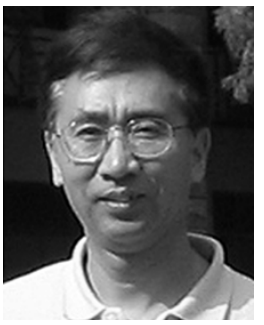

Haoping Huang received undergraduate and graduate education in geophysics at the Changchun University of Earth Sciences, Changchun, China.

$\mathrm{He}$ is currently a Research Geophysicist at Geophex, Ltd., Raleigh, NC. He was a Professor of geophysics at Changchun University of Earth Sciences from 1982 to 1992 . From 1987 to 1988 , he was a Visiting Scholar at Brown University, Providence, RI. From 1993 to 1999, he was a Research Scientist at Geoterrex-Dighem, Toronto, ON, Canada. His current research focuses on electromagnetic methods in unexploded ordnance detection and discrimination. He has published numerous research papers on electromagnetic data processing and interpretation in journals.

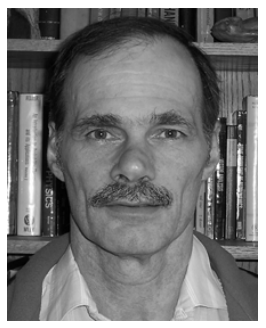

Bill SanFilipo received the B.S. degree in physics from Harvey Mudd College, Claremont, CA, the M.S. degree in applied physics (seismology) from the University of Texas at Dallas, Richardson, and the Ph.D. degree in electromagnetic geophysics from the University of Utah, Salt Lake City, in 1972, 1978, and 1984, respectively.

He has been a Research Geophysicist with Geophex, Raleight, NC, since 1998, primarily involved in EMI data analysis related to UXO and mine detection and discrimination. Prior to that he was a Software Analyst for Kearfott Guidance and Navigation Corporation (1986-1998) and a Mineral Exploration Geophysicist for CRA Exploration Pty. Ltd. in Australia (1984-1986).

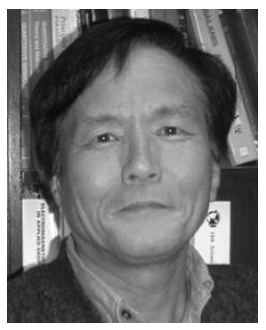

I. J. Won received the B.S. degree in mining engineering from Seoul National University, Seoul, Korea, and the M.S. and Ph.D. degrees in geophysics from Columbia University, New York, in 1967, 1971, and 1973, respectively.

He is currently President of Geophex, Raleight, $\mathrm{NC}$, an independent geophysical research and services company. From 1976 untill 1989, he was an Assistant Professor, Associate Professor, and Professor of geophysics at North Carolina State University, Raleigh. He has published over 100 research and review articles in journals and books. 